\title{
Diseño, desarrollo y evaluación de una cocina solar de bajo costo
}

\section{Design, Development and Evaluation of a Low-Cost Solar Cooking}

\author{
Patricia Llaique Herreros, Malena Salinas Portilla, Fabiola Zúñiga Ramírez, \\ Manuel Rodríguez Zúñiga*
}

http://dx.doi.org/10.21503/CienciayDesarrollo.2008.v9.08

\section{RESUMEN}

Con el conocimiento de los principios básicos de la energía solar, transferencia de calor y el acceso a materiales simples, como cartón, papel de aluminio y vidrio, se pueden construir varios modelos de cocinas y otros artefactos solares eficaces.

En líneas generales, el trabajo se basa en la divulgación, en sectores de bajos recursos económicos y en multiplicadores sociales, de los principios básicos de diseño de las cocinas solares y de la identificación de materiales comunes que pueden utilizarse en su construcción, haciendo hincapié, además, en la autoconstrucción por parte de los potenciales usuarios, para lo cual se los capacita, evitando así etapas de intermediación y comercialización.

Las crisis energéticas generalizadas y el encarecimiento gradual del acceso a fuentes convencionales, dejando de ser alcanzables para una gran franja social, obligan a optimizar los recursos técnicos e intelectuales.

Además, la enseñanza y divulgación de las cocinas solares sería una solución a las problemáticas de zonas rurales y de sectores sociales cuyos habitantes no tienen recursos para acceder a las fuentes energéticas tradicionales.

Hemos emprendido esta tarea porque estamos convencidos de que la buena formación científica, el promover la búsqueda de fenómenos en la vida cotidiana y las aplicaciones sencillas de leyes y principios son los que capacitan al niño y al joven para que puedan crear soluciones particulares frente a las necesidades y situaciones que se presentan en cada lugar, en cada oficio, trabajo o profesión.

Además, consideramos fundamental valorar e ir integrando el uso de energías alternativas en la vida de cada uno, para disminuir de esta manera el impacto medioambiental global producido.

Palabras clave: energía solar, transferencia de calor, energias alternativas.

\section{ABSTRACT}

With the knowledge of the basic principles of solar energy, heat transfer and access to simple materials such as cardboard, aluminum foil and glass, one can build several models of solar cookers and other devices effective.

Broadly, the work is based on the disclosure in areas of low economic and social multiplier of the basic principles of design of solar cookers, the identification of common materials that can be used in their construction, further emphasizing the car construction on the part of potential users, which trains them to avoid brokerage and marketing stages.

\footnotetext{
* Estudiantes del sexto semestre de la Escuela Académico-Profesional de Ingeniería Industrial de la Universidad
} Alas Peruanas. 
The generalized energy crisis and the gradual increase in access to conventional sources, no longer attainable to a wide range social, require to optimize the technical and intellectual resources.

Moreover, by teaching and dissemination of solar cookers would be a solution to the problems of rural and social sectors, whose inhabitants have no access to traditional energy sources.

We have undertaken this task because we are convinced that good science education, promoting the pursuit of phenomena in everyday life and simple applications of laws and principles, is what enables children and young people to create solutions, compared to needs and situations that arise at each location in each trade, occupation or profession.

Furthermore we consider it essential to assess and integrate the use of alternative energy in everyone's life, in this way to reduce the overall environmental impact occurred.

Key words: solar energy, heat transfer, alternative energies.

\section{INTRODUCCIÓN}

Actualmente, un tercio de la humanidad depende de la leña para cocinar. El tradicional fuego a tierra rinde alrededor de un 5\%, y el horno de leña aprovecha un máximo de $25 \%$ del calor de la madera seca. Las cocinas de gas natural o vitrocerámicas actuales permiten aprovechar hasta un $30 \%$.

Con una cocina solar podemos conseguir eficiencias de prácticamente el 50\%. Así, una actividad tan aparentemente inofensiva como cocinar los alimentos genera una importante cantidad de emisiones de gases a la atmósfera. Una familia puede consumir fácilmente una media de 4 toneladas al año de leña para cocinar, las cuales liberan alrededor de unas 7,2 toneladas de dióxido de carbono.

En términos generales, teniendo en cuenta que cerca de 2000 millones de personas cocinan con fuego a tierra y leña, en tanto que el resto lo hace con sistemas más eficientes pero que también aportan a la emisión de dióxido de carbono, podemos afirmar que alrededor de un 15\% de los 6000 millones de toneladas que liberamos a la atmósfera es producto de una actividad básica como es la de cocinar los alimentos.

Cuando hablamos de reducir las emisiones con efecto invernadero, no pensamos que nuestra vida está llena de actos cotidianos (como por ejemplo cocinar) que aportan a las emisiones.

Alrededor del 50\% de los 3200 millones de toneladas de madera recogida en todo el planeta se quema como combustible. En algunos lugares, esta proporción llega a las cuatro quintas partes. Esto significa que un producto con tantas aplicaciones tecnológicas como la madera se acaba convirtiendo en calor, como si no tuviésemos otras fuentes de calor más limpias y renovables.

Todo el mundo sabe cocinar con fuego, pero solo un $2 \%$ sabe cocinar con la energía del Sol, aunque es muy fácil hacerlo.

Hoy día, un 25\% de la humanidad carece de combustible para cocinar. Quemar combustible, además de contaminar el aire, causa la deforestación de muchos lugares donde hay una muy buena radiación solar.

\section{Objetivos}

- Contribuir al "desarrollo social sustentable" de la comunidad y formar conciencia en temas energéticos.

- Favorecer la percepción de la posibilidad y necesidad de utilizar energías alternativas en nuestras vidas. 
- Generar grupos de trabajo en sectores sociales de menores recursos, capaces de concientizarse, detectar y solucionar alguno de los problemas energéticos de su entorno, respetando siempre al medio ambiente.

- Diseñar, construir y evaluar cocinas y otros artefactos solares sencillos de bajo costo, eficientes y seguros.

- Fortalecer la integración de la Universidad Alas Peruanas con asociaciones, e intensificar su presencia en el medio.

\section{Estrategia de difusión}

El plan de difusión y promoción de la cocina solar es muy simple: hablar con tantas personas y organizaciones como sea posible, en cualquier lugar y en cualquier momento.

Estas discusiones pueden darse a diferentes niveles (3C):

\section{$\checkmark$ Concientización}

$\checkmark$ Construcción de infraestructura

$\checkmark$ Continuo seguimiento y creación de nuevas conexiones y enlaces.

Es absolutamente importante identificar una organización local líder con la cual trabajar en conjunto. También debe incluirse a las ONG’s (Organizaciones No Gubernamentales), a niveles multinacionales, nacionales, regionales, urbanos y rurales.

\section{Ámbito y alcances de la propuesta}

Está dirigido a:

- Sectores sociales marginados y de bajos recursos, que por su condición tienen difícil acceso a las fuentes tradicionales de energías debido a los altos costos relativos.

- Población general en edad escolar, para propiciar una conciencia ecológica desde edad temprana, formando de esta manera futuros divulgadores en esta temática.

\section{Desarrollo de la experiencia}

A partir de la detección de problemas energéticos de la población en general y sobre todo de la de bajos recursos, comenzamos a interrogarnos sobre la sustentabilidad del desarrollo social vinculado a la cuestión energética, y que se podría aportar en este sentido.

Considerando lo anterior, se comenzó a considerar distintas opciones, se realizó una búsqueda bibliográfica y en Internet sobre energías alternativasy formasde aprovechamiento sencillas que estén al alcance de todos los sectores sociales.

Nos decidimos por la utilización de la energía solar, debido a que es la energía alternativa más fácil de obtener y de aprovechar.

Los datos obtenidos de la búsqueda fueron analizados y procesados desde el punto de vista de la ingeniería y de su aplicabilidad práctica, teniendo en cuenta conceptos termodinámicos para evaluar los diferentes diseños y justificar los principios de funcionamiento.

Se construyeron algunos modelos propuestos por la bibliografía y otros que fueron rediseñados, tratando de mejorar su eficiencia o disminuir su costo de fabricación. Se buscó en todos los casos cumplir con los siguientes requisitos:

- Construcción de bajo costo, con materiales como cartón, poliuretano expandido, papel aluminio, vidrio, madera, alambres, chapas. Incluso se consideró la posibilidad de utilizar materiales de descarte.

- Construcción sencilla, para que pueda ser realizado por cualquier persona, y así se multiplique su uso. 
- Que funcione y solucione en parte los problemas de pasteurización del agua y los de cocción de comidas sencillas.

\section{Localización física o geográfica}

De acuerdo a lo previsto se decidió dar inicio a las actividades de las cocinas solares, en beneficio de las zonas más pobres del distrito de Cerro Colorado (Cono Norte)

\section{CALENDARIO DE ACTIVIDADES}

\begin{tabular}{|c|c|c|c|}
\hline ACTIVIDAD & Octubre & Noviembre & Diciembre \\
\hline $\begin{array}{l}\text { Revisión de } \\
\text { información }\end{array}$ & $X$ & & \\
\hline $\begin{array}{l}\text { Construcción de la } \\
\text { cocina }\end{array}$ & & $X$ & \\
\hline $\begin{array}{l}\text { Preparación para la } \\
\text { recolección de datos }\end{array}$ & & $\mathrm{X}$ & \\
\hline $\begin{array}{l}\text { Recolección de } \\
\text { datos }\end{array}$ & & & $\mathrm{X}$ \\
\hline $\begin{array}{l}\text { Procesamiento de } \\
\text { datos }\end{array}$ & & & $\mathrm{X}$ \\
\hline $\begin{array}{l}\text { Análisis e } \\
\text { interpretación de } \\
\text { datos }\end{array}$ & & & $X$ \\
\hline $\begin{array}{l}\text { Informe final y } \\
\text { entrega del trabajo }\end{array}$ & & & $X$ \\
\hline
\end{tabular}

\section{MATERIAL Y MÉTODO}

La cocina que vamos a presentar es de tipo horno. Existen tres tipos de cocinas solares, la de tipo horno, las que concentran la radiación solar y las de tipo panel.

Los usuarios en general y nosotros preferimos las de tipo horno, pues se simplifica mucho el trabajo de cocinar al sol.

Para que la cocina sea exitosa, es indispensable cuidar los detalles. Una correcta atención a los detalles hace que la cocina funcione bien. En cambio, una realización descuidada va a significar que la cocina funcionará mal o no funcionará.
El diseño que vamos a dar es el básico, con la posibilidad de ser mejorado. Además, indicamos algunos aspectos en los cuales se puede mejorar.

La cocina tiene las siguientes partes fundamentales: el fondo, los lados, la parte anterior (delantera), la parte posterior (trasera), la tapa, la cubierta de vidrio y las aletas reflectoras.

Todas las piezas deben ser hechas de perfiles de madera, rellenados totalmente con el aislante,

\section{Materiales}

$\checkmark$ Triplay para las cajas interior y exterior.

$\checkmark$ De 6 a 8 placas de aluminio.

$\checkmark$ Un tarro pequeño de pintura negra mate (sin plomo) o ceniza de madera limpia.

$\checkmark$ Por lo menos medio litro de goma blanca o goma de harina.

$\checkmark$ Una plancha de vidrio del tamaño de la caja exterior o al menos $2 \mathrm{~cm}$ más grande que la caja interior.

Al seleccionarse los materiales, se debe tener en cuenta la resistencia a la humedad; por lo tanto, los materiales que se utilizan típicamente en la construcción de las cocinas solares, fundamentalmente son tres (material para estructura, material transparente y los aislantes).

\section{DISEÑO Y PROPORCIONES}

\section{Tamaño de la caja}

El tamaño de la caja debe estar en relación con el peso, estructura de aislamiento y resistencia a la humedad

Una cocina solar debe clasificarse según el tamaño, tomando en consideración los siguientes factores: 
- El tamaño debe permitir sostener el o los recipientes de capacidad igual o mayor a los usados normalmente para la cocción de los alimentos

- El diseño de la caja debe adaptarse a los materiales de que se dispone en el lugar de uso.

- El diseño debe permitir la correcta incidencia de energía luminosa sobre los vidrios y placas de aluminio.

- Si la cocina solar requiere continuo traslado, debe considerarse un tamaño moderado para evitar dificultades en el traslado

\section{Ventajas}

- Su fabricación es de materiales inofensivos para el medio ambiente.

- Permanecen y soportan durante todo el día una temperatura interior de hasta $200{ }^{\circ} \mathrm{C}$, sin emisión de gases y vapor.

- Exteriormente, soportan sin problema prolongadas temperaturas.

- Con su reducido tamaño y peso no ocupan mucho espacio.

- Tienen un excelente comportamiento con el calor, ya que el interior se encuentra herméticamente cerrado, de tal manera que se minimizan corrientes de aire frío.

- Su rendimiento es muy similar al de una cocina convencional.

- Pueden ser usadas para cocinar y/o hornear alimentos, preparar comidas calientes y secar frutos.

- Permite mantener la comida caliente hasta por 3 horas.

- Los alimentos pierden menos vitaminas, ya que necesitan menos agua (aprox. 2/3 menos) para su cocción.

- No requiere cuidados especiales; se cocina sin preocuparse de la ocurrencia de algún accidente
Se aprovecha la energía no convencional y reduce de manera significativa la emisión de gases tóxicos al medio ambiente, contribuyendo así a disminuir el efecto invernadero.

\section{ETAPAS DE CONSTRUCCIÓN}

$>$ I ETAPA: placa

II ETAPA: armazón del reflector

$>$ III ETAPA: caja externa

IV ETAPA: caja interna

V ETAPA: colocación del aislante

$>$ VI ETAPA: unión de piezas

$>$ VII ETAPA: acabado

\section{Construccción}

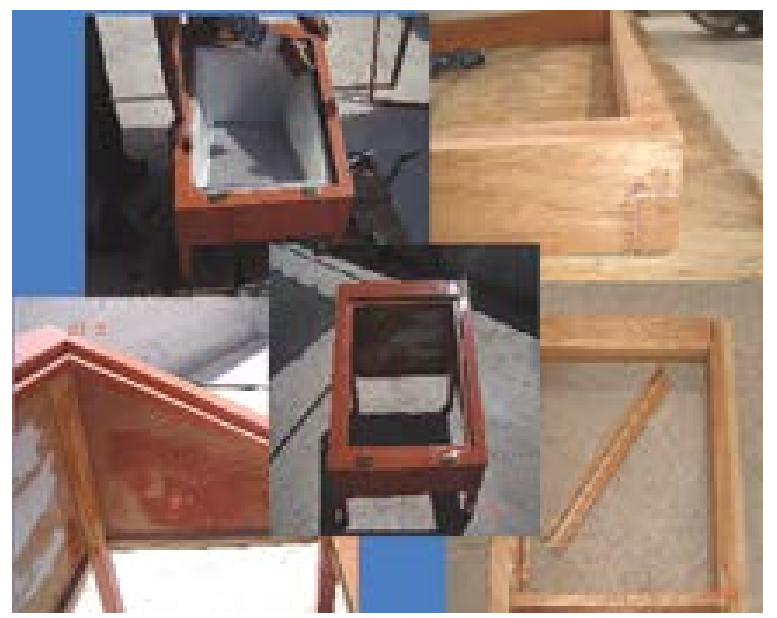

Figura 1. Elementos de una cocina solar

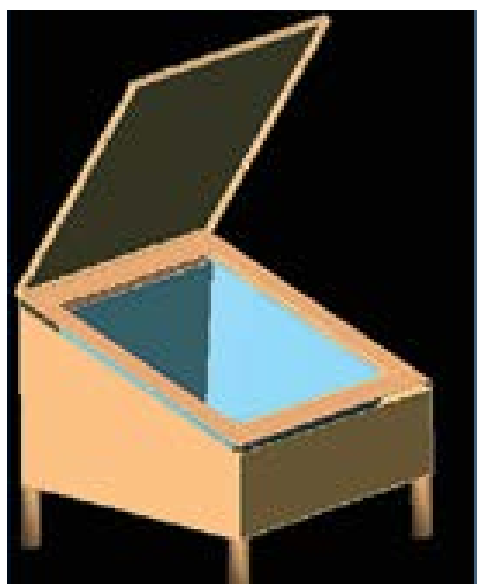

Figura 2. Esquema de cocina solar 


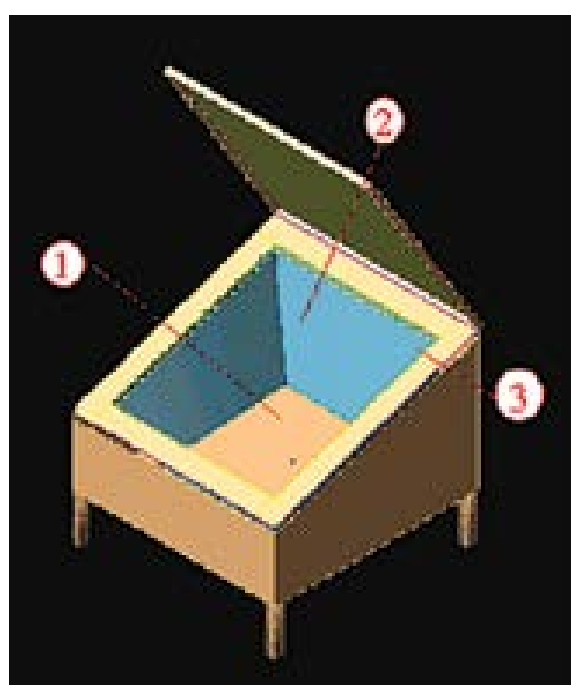

Figura 3. Partes de una cocina solar terminada

\section{PRUEBAS EXPERIMENTALES}

Prueba en vacío

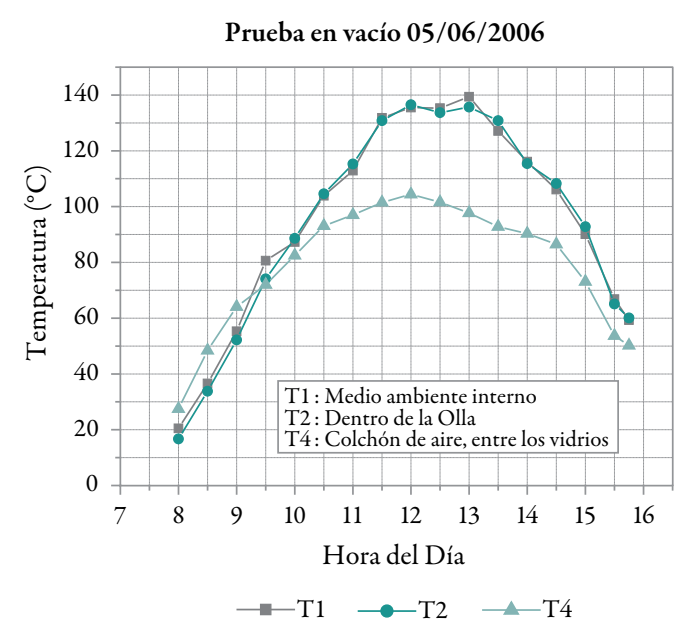

Figura 5

\section{Interpretación:}

En la figura 5 se muestran las temperaturas de los 3 termopares colocados en la cocina solar cuandoesta nocontiene un elemento para cocción en su interior. Podemos ver que la temperatura interna $(T-1)$ y la expresada en el termopar 2 (temperatura del producto) son muy similares, mientras que en el termopar 4 (temperatura

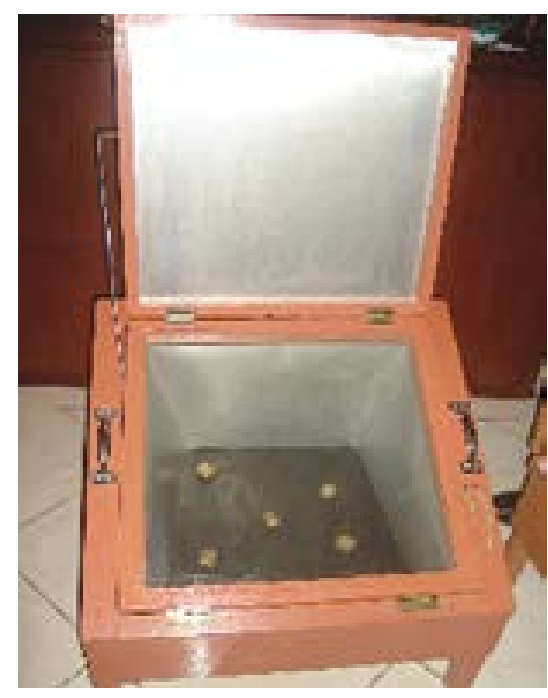

Figura 4. Cocina solar terminada

interna entre los vidrios) es de $35^{\circ} \mathrm{C}$ menor a las temperaturas antes descritas.

Esta diferencia podría deberse a factores climatológicos tales como viento, cantidad de nubes, sombra, entre otros.

No obstante, se observa que las tres temperaturas son crecientes hasta alcanzar su máximo (aproximadamente a la 13:00 h), a partir del cual las temperaturas descienden, observándose para este caso una curva simétrica.

Prueba en arroz: temperatura en el producto

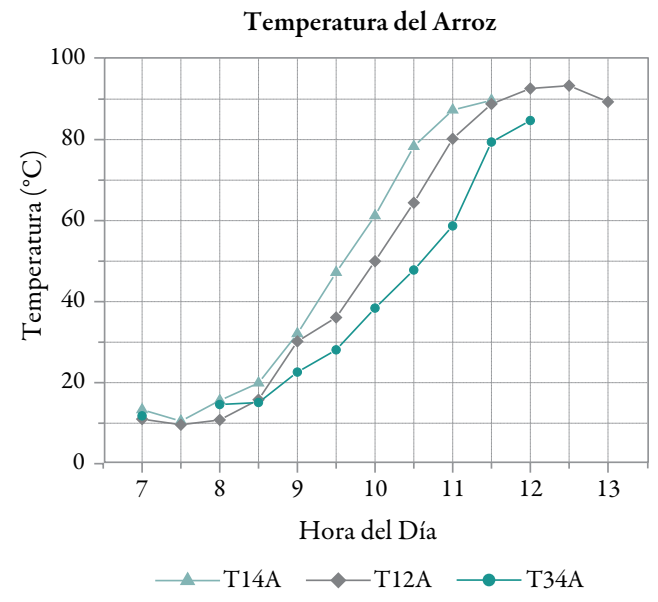

Figura 6 


\section{Interpretación:}

En la figura 6 podemos ver las diferentes curvas que toman las temperaturas para $1 / 4,1 / 2 y$ $3 / 4$ de $\mathrm{kg}$ de arroz, las cuales fueron medidas desde las 7:00 horas, alcanzando su máxima al medio día.

Las temperaturas del producto siguen un recorrido creciente, pudiéndose observar que para $1 / 4$ de $\mathrm{kg}$ la temperatura se eleva con mayor rapidez a las 11:50 h, en comparación con las otras dos pruebas; esto se debe a la proporción inversa entre volumen y la temperatura del producto.

Para los casos de $1 / 2, y 3 / 4$, las temperaturas se igualan a las 12:00 horas, por lo que se podría decir que la cantidad del producto (volumen) es inversamente proporcional a la temperatura, pero esta es directamente proporcional con el tiempo, pudiendo variar en algunos casos ligeramente según la proporción de agua en relación al volumen del producto.

\section{A MENOR VOLUMEN, MAYOR TEMPERATURA Y MENOR TIEMPO DE COCCIÓN}

Prueba en papa - temperatura en el producto

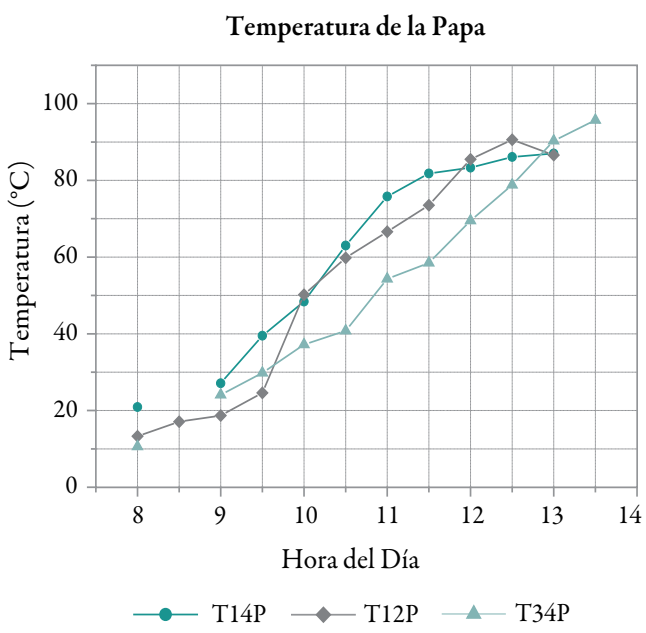

Figura 7

\section{Interpretación:}

La figura 7 describelastemperaturas realizadas para la papa de 1/4, 1/2 y 3/4 de kilogramo, medidas a partir de las 08: $00 \mathrm{~h}$. hasta la 13:30 h.

Las tres temperaturas registradas presentan un comportamiento creciente, no siguiendo una relación homogénea con los otros productos (por ejemplo, arroz). En ciertos intervalos de tiempo, se observa que a mayor volumen la temperatura es menor, con una diferencia aproximadamente de $20^{\circ} \mathrm{C}$ entre los tres productos. Así mismo, se observa que los tres productos a la misma hora elevan la temperatura.

Esta variación podría deberse a factores externos espontáneos, como el viento, cantidad de nubes, la sombra, entre otros.

El día 16 de diciembre del 2007 (se puso 1/2 $\mathrm{kg}$ de papa), el medio ambiente externo varió constantemente, afectando sobremanera las temperaturas medidas.

A consecuencia de estos cambios, la temperatura interna del producto se incrementó en $30{ }^{\circ} \mathrm{C}$ repentinamente, lo cual ocasionó la ebullición en el agua que contenía el producto.

Prueba en arroz - medio ambiente interno en la cocina

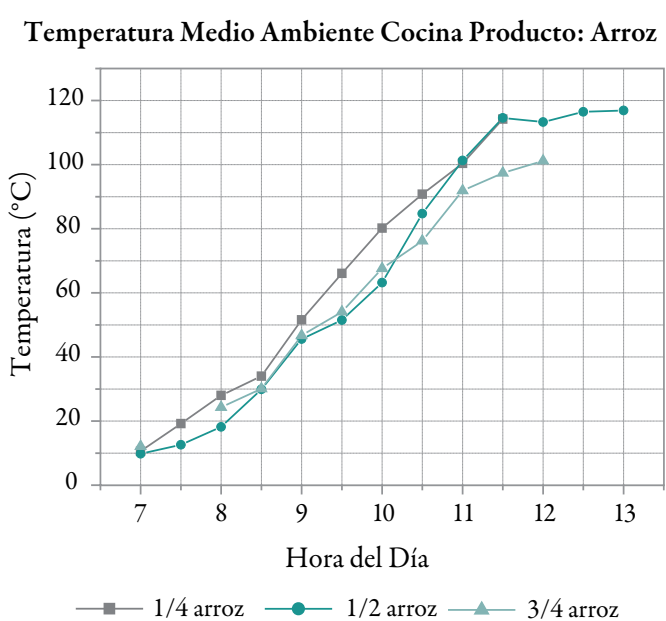

Figura 8 


\section{Interpretación:}

La figura 8 representa las temperaturas para $1 / 4,1 / 2$ y $3 / 4$ de kilogramo de arroz respectivamente, tomadas a partir de las 7:00 h a las $13.00 \mathrm{~h}$, hora en la cual se llevó a cabo la cocción del arroz.

El comportamiento de las temperaturas es creciente simultáneamente hasta que alcanza su punto máximo a las $12: 30 \mathrm{~h}$, a partir de la cual muestran un comportamiento lineal, siempre existiendo una diferencia entre ellas. Cabe resaltar que a ciertos intervalos las diferencias de temperatura no son muy marcadas.

La relación entre volumen, temperatura y tiempo del producto no es proporcional.

\section{A MENOR VOLUMEN, MAYOR TEMPERATURA Y MENOR TIEMPO DE COCCIÓN}

Esta diferencia podría deberse a factores climatológicos externos espontáneos.

Es preciso mencionar que el 19 de diciembre (fecha en la que se sometió a cocción $1 \frac{1}{2}$ kilogramo de arroz), a las 13 horas, alcanzó su máxima temperaturas $\left(116,9^{\circ} \mathrm{C}\right)$, presentando un clima favorable; sin embargo, la cantidad de agua suministrada $(535 \mathrm{ml})$ al producto no fue suficiente, provocando la sobrecocción del mismo.

\section{Prueba en carne, papa y arroz}

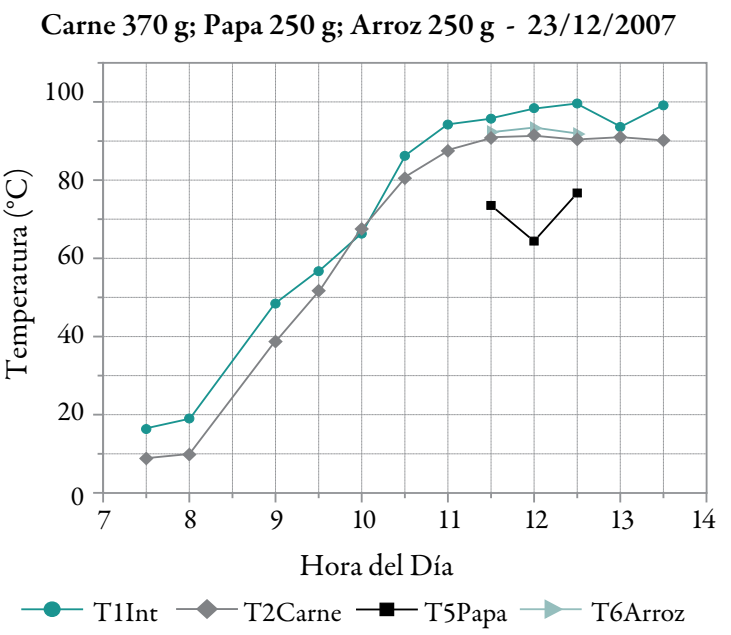

\section{Interpretación:}

La figura 9 muestra la relación de cocción en tres productos (carne, papa y arroz) estudiados a partir de las 7:30 h hasta las 13:30 h, utilizando para ello cuatro termopares distribuidos de la siguiente manera:

$\mathrm{T}-1$ : Temperatura o ambiente interno de la cocina.

$\mathrm{T}-2$ : Temperatura del producto (carne).

$T$ - 5: Temperatura del producto (papa).

$\mathrm{T}$ - 6: Temperatura del producto (arroz).

Los tres productos presentan un comportamiento creciente con una variación máxima de $10^{\circ} \mathrm{C}$ entre cada registro de medida.

Sin embargo, el tiempo de cocción para cada producto fue distinto, debiéndose tal vez a que la proporción entre producto y agua no fue la correcta.

\section{CONCLUSIONES}

El uso de la cocina solar es posible en cualquier lugar donde exista una buena incidencia solar.

En condiciones óptimas de clima, hay una relación inversa entre volumen y temperatura; por consiguiente, a mayor volumen, mayor temperatura y menor tiempo de cocción; por lo tanto, el tiempo de cocción depende del volumen.

Las condiciones climáticas como viento y nubosidad pueden variar únicamente el tiempo de cocción.

El tiempo de cocción para cada producto es distinto cuando la proporción entre producto y agua no es bien balanceada 
Al someter a cocción dos o tres productos de naturaleza diferente, las temperaturas presentan un comportamiento creciente.

En una cocina solar se puede preparar cualquier tipo de alimentos, inclusive tortas, a excepción de frituras y hervido de leche.

Para producir un impacto real en la sociedad en lo referente a este tema, es necesario conseguir un cambio de mentalidad en las personas en la forma de encarar los problemas energéticos así como formular proyectos que orienten la manera de encarar la problemática de la energética global.

Es importante que personas con una instrucción bastante sólida, y aquellas con escasa o nula formación científica, se muestren interesadas en la construcción de cocinas solares con pocos recursos, aprovechando eficientemente una fuente inagotable de energía solar. 